\title{
HmmPfam Algorithm
}

National Cancer Institute

\section{Source}

National Cancer Institute. HmmPfam Algorithm. NCI Thesaurus. Code C49045.

A program that searches Hidden Markov Models from the PFAM database for significantly similar sequence matches to the input protein sequence. 